\title{
Homogénéité des Filaires de Batraciens des genres Waltonella, Ocboterenella et Madochotera; création des Waltonellinae n. subfam.
}

\author{
par O. BAIN et J. PROD'HON \\ Laboratoire de Zoologie (Vers) associé au C.N.R.S. (P ${ }^{\mathrm{r}}$ A.-G. Chabaud), \\ Muséum national d'Histoire naturelle, 43, rue Cuvier, F 75231 Paris Cedex 05 \\ et Entomologie médicale, O.R.S.T.O.M., F 93140 Bondy
}

\section{Résumé.}

Description de 3 nouvelles Filaires de Batraciens malgaches et sud-américains : Madochotera pichoni, n. sp., Ochoterenella guibei, n. sp. et Waltonella guyanensis n. sp.

Les descriptions montrent que les Filaires de Batraciens, autres que les Icosiellinae Anderson, 1958, qui étaient dispersées dans 2 sous-familles, les Onchocercinae (avec Ochoterenella Caballero, 1944 et Madochotera Bain et Brunhes, 1968) et les Dirofilariinae (avec le sous-genre Waltonella Schacher, 1974, groupant les Foleyella de Batraciens, à l'exclusion de ceux de Reptiles), forment un groupe homogène et original qui justifie la création d'une nouvelle sous-famille, Waltonellinae $\mathrm{n}$. subfam.

\section{Summary.}

Homogeneity of the Filariae of Batrachians, including the genus Waltonella, Ochoterenella and Madochotera; creation of Waltonellinae n. subfam.

Description of three new Filariae of Madagascar and South American Batrachians: Madochotera pichoni n. sp., Ochoterenella guibei, n. sp. and Waltonella guyanensis $\mathrm{n}$. sp.

These descriptions show that the Filariae of Batrachians other than the Icosiellinae Anderson, 1956, which belonged to two subfamilies, Onchocercinae (with Ochoterenella Caballero, 1944 and Madochotera Bain et Brunhes, 1968) and Dirofilariinae (with the subgenus Waltonella Schacher, 1974, including the Foleyella of Batrachians, except those of Reptiles) consist in an homogeneous and original group which justifies the creation of a new subfamily, Waltonellinae n. subfam. 


\section{Introduction}

Les Filaires de Batraciens, autres que les Icosiellinae Anderson, 1958, sont actuellement réparties dans 3 genres, Foleyella Seurat, 1917 (= Foleyellides Caballero, 1935) (1), Ochoterenella Caballero, 1944 et Madochotera Bain et Brunhes, 1968, et appartiennent à 2 sous-familles, Dirofilariinae et Onchocercinae.

Foleyella est placé dans les Dirofilariinae en raison de la présence d'ailes caudales bien développées chez le mâle (cf. Chabaud et Anderson, 1959). Schacher et Crans (1973) ont montré que ce genre comprenait 2 ensembles morphologiquement bien distincts : le sous-genre Waltonella Schacher 1974 (=Waltonia Schacher et Crans, 1973, préemployé) qui groupe les Filaires de Batraciens et le sous-genre Foleyella, uniquement parasite de Reptiles.

Ochoterenella est décrit comme distinct de Foleyella par l'absence d'ailes latérales, la structure des spicules et l'absence de gaine de la microfilaire. Chabaud et Anderson (1959), se basant sur l'absence d'ailes latérales, placent ce genre dans les Onchocercinae.

Madochotera est proche d'Ochoterenella et placé également dans les Onchocercinae, malgré l'existence de petites ailes latérales.

Il apparaît donc que la présence, ou l'absence, d'ailes latérales ne justifie pas la dispersion, dans 2 sous-familles distinctes, de ces genres et sous-genre, dont l'organisation générale est homogène : œsophage glandulaire bien développé, queue longue et sans pointe, files submédianes de grosses papilles cloacales non pédonculées, spicules dissemblables et simples, vulve nettement pré-équatoriale. Les affinités entre ces genres sont encore renforcées par les récentes données sur les structures céphaliques de quelques espèces; la description de $F$. (Waltonella) flexicauda (Schacher et Crans, 1973), celle de $F$. (W.) confusa Schmidt et Kuntz, 1969, la redescription précise (Anderson, 1968) de la tête de $F$. (W.) dolichoptera (Wehr et Causey, 1939) et d'Ochoterenella digiticauda Caballero, 1944, et les descriptions des Madochotera (cf. Bain et Brunhes, 1968 et Prod'hon et Bain, 1973) montrent que toutes ces espèces ont un plateau céphalique rectangulaire, très étiré latéralement, dont les angles sont soutenus par 4 papilles céphaliques volumineuses; en outre, à l'exception d' $O$. digiticauda, elles portent 2 expansions cuticulaires saillantes parabuccales, toujours situées dans le plan latéral.

L'impression qui se dégage donc actuellement des descriptions précises de ces Filaires de Batraciens est que le sous-genre Waltonella, qui a peu de traits communs avec le sous-genre Foleyella, constitue avec Ochoterenella et Madochotera un groupe original et homogène.

La description que nous donnons de 3 nouvelles Filaires de Batraciens, 2 d'origine malgache et une provenant d'Amérique du Sud, vient étayer cette notion, en montrant principalement que certains caractères particuliers, généralement passés

(1) Classification de Chabaud et Anderson, 1959. 
inaperçus comme le plateau céphalique étiré latéralement et l'area rugosa, constituent des traits constants et originaux de ces Filaires; elles semblent nécessiter la création d'une nouvelle sous-famille.

A l'intérieur de ce groupe, la variation de quelques caractères qui nous paraissent importants, comme la position de la vulve et la présence ou l'absence des formations cuticulaires parabuccales, justifie des divisions génériques qui permettent de conserver les genres (ou ancien sous-genre) précédemment créés.

Les Filaires, dont nous donnons la description, sont conservées au Muséum National d'Histoire Naturelle de Paris.

\section{Madochotera pichoni n. sp.}

Hôte: Racophorus sp., Racophoridae.

LOCALISATION: cavité générale.

Provenance: Andringitra, Madagascar (référence de récolte: Jonah 5-XII).

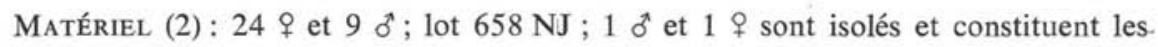
types.

\section{Description.}

Filaires de taille moyenne, assez larges, un peu amincies dans la région antérieure; cuticule ornée, mais différemment dans les 2 sexes; ailes latérales allant presque jusqu'aux extrémités du corps. Plateau céphalique rectangulaire avec papilles. disposées suivant la figure $1 \mathrm{~B}$ et $2 \mathrm{~B} ; 2$ saillies cuticulaires latérales bordent la bouche; capsule buccale tubulaire, peu cuticularisée et courte. Esophage formé d'une courte portion musculaire et d'une longue portion glandulaire, légèrement plus large (fig. $1 \mathrm{~A}$ et $2 \mathrm{~A}$ ). Intestin large. Pas de pointes caudales.

FEMELle (fig. 1): cuticule ornée de sillons transversaux régulièrement espacés (fig. $1 \mathrm{G}$ ). Ailes latérales soutenues dans la région antérieure par une structure fibrillaire très nette (fig. $1 \mathrm{C}$ ). Vulve très éloignée de la tête; le corps se renfle ventralement en arrière de l'orifice vulvaire; portion impaire de l'ovéjecteur de forme simple sans sphincter différencié (fig. $1 \mathrm{H}$ et I), orientée vers l'avant ou vers l'arrière du corps. Ovaires situés en avant de la vulve; extrémité distale des ovaires conique (fig. $1 \mathrm{~J}$ ). Rectum étroit et allongé. Queue très longue, qui s'amenuise régulièrement vers l'extrémité caudale obtuse, à cuticule très épaisse (fig. $1 \mathrm{D}$ et $\mathrm{E}$ ).

Dimensions: + type : corps long de $2,78 \mathrm{~cm}$ et large de $310 \mu$; ailes latérales naissant à 35 et $17 \mu$. de la tête et se terminant à $45 \mu$ de l'extrémité caudale. Plateau céphalique mesurant $35 \mu . / 22 \mu$. Capsule buccale haute de $7 \mu$; anneau nerveux à $230 \mu$ de la :ê e ;

(2) Nous remercions très vivement le $D^{r}$ E. R. Brygoo, Directeur de l'Institut Pasteur deMadagascar, qui nous a confié ce matériel. 

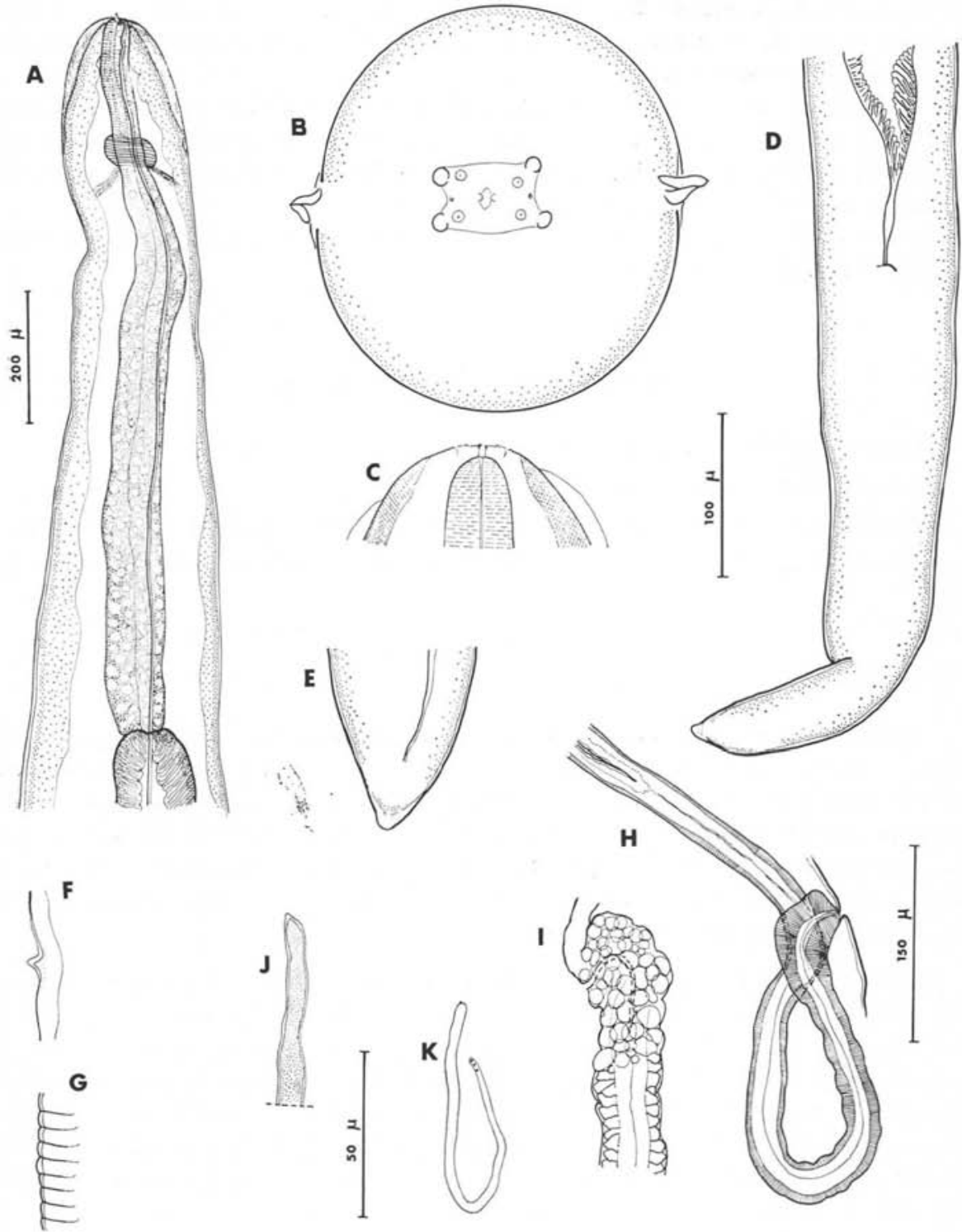

FIG. 1. - Madochotera pichoni n.sp. १. A) Région antérieure, vue latérale. B) Tête, vue apicale. C) Idem, vue latérale. D) Queue, vue ventrale. E) Extrémité caudale, vue latérale. F) Aile latérale en section transversale à mi-hauteur du corps. G) Cuticule du corps ornée de sillons transversaux. H) Portion impaire de l'ovéjecteur. I) Musculature de la région vaginale, après dissection. J) Apex d'un ovaire. K) Microfilaire prélevée dans l'utérus. (A, D et $\mathrm{I}$ : éch. $200 \mu ; \mathrm{B}, \mathrm{F}$ et $\mathrm{K}$ : éch. $50 \mu ; \mathrm{C}, \mathrm{E}, \mathrm{G}$ et $\mathrm{J}$ : éch. $100 \mu ; \mathrm{H}$ : éch. $150 \mu$ ). 


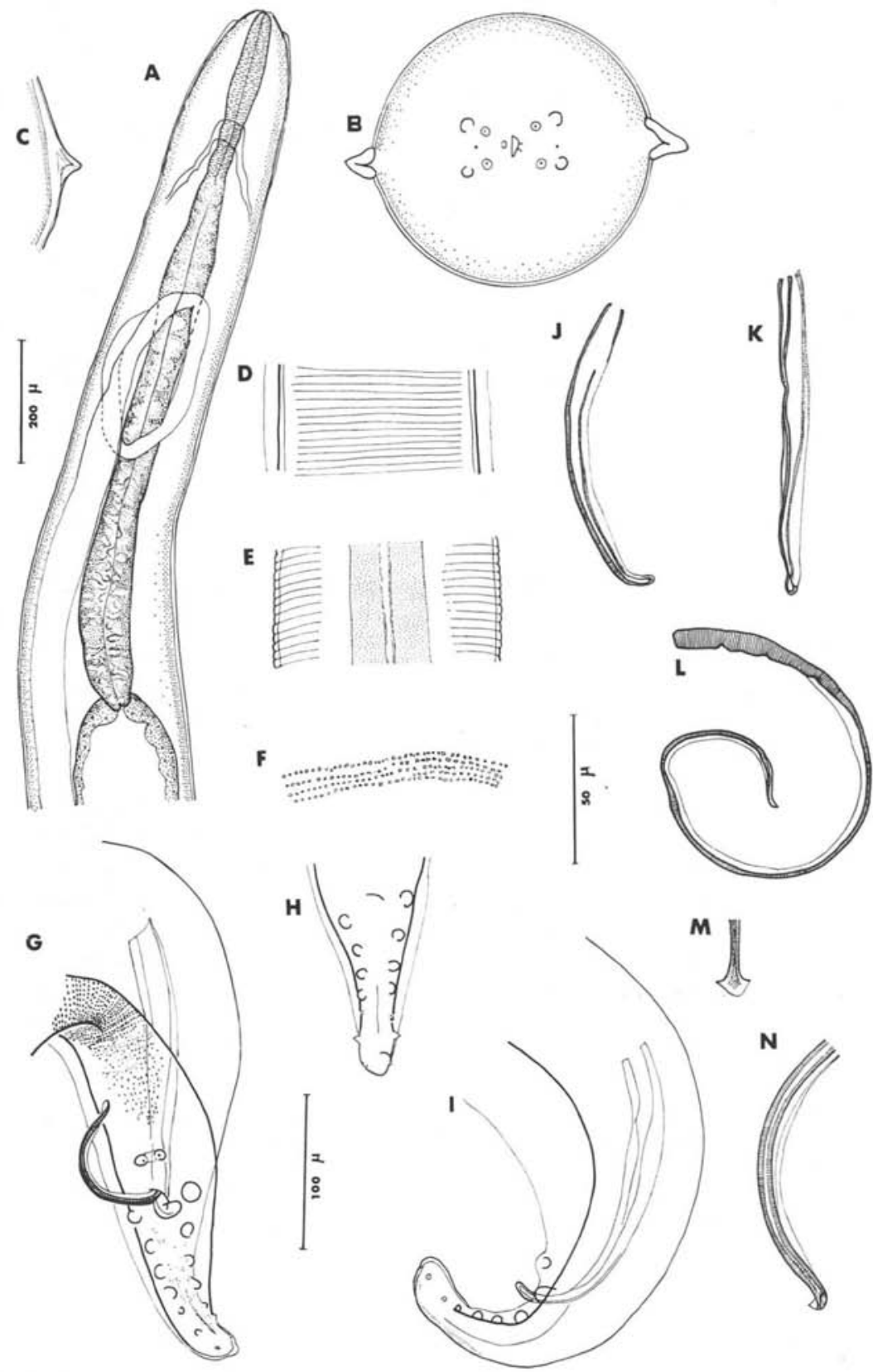

FIG. 2. - Madochotera pichoni n.sp. ô. A) Région antérieure, vue médiane. B) Tête, vue apicale. C) Aile latérale, en section transversale, à mi-hauteur du corps. D) Ornementation cuticulaire de la région moyenne du corps, vue médiane. E) Idem, vue latérale. F) Area rugosa $150 \mu$ en avant du cloaque, vue ventrale. G) Région caudale, vue semi-ventrale. $\mathrm{H}$ ) Queue, vue ventrale. I) Région caudale, vue latérale. J) Spicule droit disséqué, vue latérale droite. K) Idem, vue ventrale. L) Spicule gauche, vue latérale. M) Extrémité distale du spicule gauche, vue ventrale. N) Région distale du spicule gauche, vue latérale. (A: éch. $200 \mu$; B, C, M et $\mathrm{N}$ : éch. $50 \mu ; \mathrm{D}, \mathrm{E}, \mathrm{F}, \mathrm{G}, \mathrm{H}, \mathrm{I}, \mathrm{J}, \mathrm{K}$ et L: éch. $100 \mu$ ). 
œsophages musculaire et glandulaire longs respectivement de 225 et $870 \mu$; vulve à $4900 \mu$ de la tête; ovéjecteur impair long de $1700 \mu$; queue longue de $950 \mu$.

- Pour les autres $q$, corps long de 2,7 à $3,6 \mathrm{~cm}$; vulve distante de 5200 à $8570 \mu$ de la tête ; queue longue de 750 à $1600 \mu$.

MÂle (fig. 2) : région caudale large et enroulée sur 2 à 3 tours de spires; corps localement aminci juste en avant de cette région. Cuticule du corps ornée de sillons transversaux plus marqués que chez la femelle et qui s'interrompent à quelque distance des champs latéraux (fig. 2 E). Les sillons dorsaux s'interrompent dans la région postérieure enroulée; les sillons ventraux s'arrêtent un peu en avant pour faire place à une autre ornementation qui constitue l'area rugosa; elle est formée de rangées transversales de pointes cuticulaires cunéiformes qui ne s'interrompent qu'en avant du cloaque; la queue elle-même n'est ornée ventralement que de très fines stries transversales; la face dorsale de la pointe caudale est également finement striée (fig. $2 \mathrm{~F}$ à I). Papilles caudales disposées suivant la figure $2 \mathrm{G}$ et $\mathrm{H}$; papille préanale dédoublée; les 2 paires de papilles postanales les plus postérieures sont latérales, les autres sont latéro-ventrales. Spicule gauche avec manche court et lame mince et longue bordée par 2 ailes étroites et terminé par une languette lamellaire losangique (fig. $2 \mathrm{~L}, \mathrm{M}$ et $\mathrm{N}$ ). Spicule droit courbé ventralement, assez large, bordé par 2 ailes étroites, à extrémité arrondie (fig. $2 \mathrm{~J}$ et $\mathrm{K}$ ). Queue brusquement amincie, puis légèrement renflée à son extrémité, qui est arrondie (fig. $2 \mathrm{G}$ et I).

Dimensions : $\hat{\delta}$ type : corps long de $1,36 \mathrm{~cm}$ et large de $300 \mu(130 \mu$ de large juste en avant de la région enroulée); ailes latérales naissant à $28 \mu$ de la tête et se terminant à $40 \mu$ de la queue; naissance des sillons transversaux à $1400 \mu$ de la tête; fin des sillons dorsaux à $1700 \mu$ de la pointe caudale ; area rugosa remontant $2750 \mu$ en avant du cloaque ; anneau nerveux à $210 \mu$ de la tête ; capsule buccale haute de $7 \mu$; œsophages musculaire et glandulaire longs respectivement de 290 et $840 \mu$; spicules droit et gauche longs respectivement de $218 \mu$ et $508 \mu$ (manche long de $100 \mu$ ); queue longue de $115 \mu$.

- Pour les autres $\hat{\delta}$, corps long de 1,27 à $1,36 \mathrm{~cm}$, large de 220 à $255 \mu$ avec œsophage long de 950 à $1050 \mu$.

Microfilaires (fig. $1 \mathrm{~K}$ ) (extraites de l'utérus) : corps long de $118 \mu$ sur $3 \mu$ de large. Tête ronde ornée d'un petit crochet subapical; largeur maximum du corps atteinte dans la région antérieure; moitié postérieure régulièrement amincie ; extrémité caudale obtuse.

\section{Discussion.}

L'œsophage glandulaire bien développé, le plateau céphalique rectangulaire étiré latéralement, la capsule buccale présente, bien que peu cuticularisée, les papilles caudales non pédonculées et l'area rugosa, opposent nos spécimens aux 4 Foleyella de Reptiles : F. candezei (Fraipont, 1882) en Afrique, et F. furcata (Linstow, 1899), F. brevicauda Chabaud, Anderson et Brygoo, 1959 et F. b. magnilarvatum Bain, 1968 à Madagascar. 
L'analogie de nos spécimens avec les Filaires de Batraciens, Waltonella, Madochotera et Ochoterenella est évidente en ce qui concerne les structures de l'œsophage, de la région caudale du mâle et des spicules. En outre, chez les 6 espèces où la structure céphalique est connue, la tête forme un plateau étiré latéralement et est le plus souvent ornée de 2 expansions cuticulaires parabuccales latérales.

Parmi les nombreuses Filaires de Batraciens, nos spécimens se rapprochent plus particulièrement des Madochotera, tous malgaches, par la présence des expansions parabuccales, les papilles céphaliques sans pointe articulée et la position de la vulve qui est nettement postérieure à la fin de l'œsophage.

Les 3 espèces déjà connues sont également parasites de Racophoridae, mais se distinguent toutefois de nos spécimens par de nombreux caractères:

- M. alata Bain et Brunhes, 1968, décrit à partir d'un seul mâle, a un plateau céphalique plus grand, des ailes latérales plus réduites à leur naissance, un œsophage glandulaire plus long, une queue nettement plus courte, une area rugosa constituée de crêtes allongées et pas d'ornementation cuticulaire sur le reste du corps.

- M. landauae Prod'hon et Bain, 1973, a un œsophage glandulaire dissymétrique à sa naissance, des pointes cuticulaires parabuccales plus éloignées de la bouche ; chez le mâle, la cuticule du corps est ornée de bandes transversales ponctuées, les ailes latérales se terminent plus près du cloaque, l'area rugosa est constituée de crêtes allongées, le spicule gauche a des ailes plus larges, le spicule droit est plus court ; chez la femelle, la queue est plus courte et de forme différente, la microfilaire est plus large et se rétrécit brusquement dans la région caudale.

- Madochotera sp. Bain et Brunhes, 1968, connue seulement par une femelle, a un œsophage glandulaire plus long, une microfilaire plus large et plus courte, amincie seulement dans la région caudale.

Nos Filaires constituent donc une nouvelle espèce, Madochotera pichoni n. sp.

\section{Ochoterenella guibei n. sp.}

HôTE: Mantidactylus redimitus (Btgr), Racophoridae.

LOCALISATION: cavité générale.

Provenance : Marojezy (300 m d'altitude), Madagascar.

Matériel : 6 \%, 4 ठै (3); lot type 814 NJ.

\section{Description.}

Filaires courtes et très larges, brusquement amincies au niveau de l'œsophage musculaire et après l'anus. Pas d'ailes latérales. Plateau céphalique rectangulaire,

(3) Nous remercions très vivement le Pr J. Guibé, du Muséum National d'Histoire Naturelle qui nous a confié ce matériel.

Annales de Parasitologie humaine et comparée (Paris), t. $49, \mathrm{n}^{\circ} 6$ 
souvent relevé sur ses bords latéraux, avec papilles disposées plus ou moins régulièrement ; papilles céphaliques comportant une base large et un segment apical saillant (fig. $3 \mathrm{C}$ et $\mathrm{D}$ et $4 \mathrm{C}$ et $\mathrm{D}$ ). Bouche plus ou moins arrondie. Capsule buccale à section transversale triangulaire, courte, large, à paroi nettement cuticularisée ; elle est souvent renforcée sur les parois latéroventrales par une mince couche cuticulaire très réfringente qui affleure sous le tégument céphalique; parfois, seuls les angles latéraux sont renforcés, mais ils ne font pas saillie à la surface. Esophage avec portion musculaire courte et portion glandulaire épaisse et développée. Intestin très large. Pas de pointes caudales.

Femelle (fig. 3): cuticule du corps ornée ventralement et dorsalement de rides transversales discontinues et bien saillantes qui débutent un peu en arrière de l'œsophage (fig. $3 \mathrm{E}$ et $\mathrm{H}$ ). Vulve un peu en arrière ou au niveau de l'œsophage, grande et fortement déprimée. Ovéjecteur simple, dépourvu de sphincter; portion impaire longue ; musculature vulvaire très développée (fig. $3 \mathrm{~A}, \mathrm{~F}$ et $\mathrm{G}$ ). Utérus minces enroulés en boucles obliques. Rectum allongé. Queue conique.

Dimensions: Corps long de $1,64 \mathrm{~cm}$ et large de $820 \mu$; capsule buccale haute de $8 \mu$; plateau céphalique de $30-42 \mu / 35 \mu$; anneau nerveux à $220 \mu$ de l'apex ; œsophages muslaire et glandulaire longs respectivement de 250 et $950 \mu$; vulve à $1350 \mu$ de l'apex ; portion impaire de l'ovéjecteur longue de $2,1 \mathrm{~mm}$.

-2 autres $q$ : corps longs de 1,94 et $2,14 \mathrm{~cm}$ et larges de 700 et $730 \mu$; œsophage glandulaire long de 1070 et $1200 \mu$; vulve à 850 et $1500 \mu$ de l'apex; queue longue de $240 \mu$.

MÂle (fig. 4) : naissance du testicule un peu en arrière de l'œsophage; il remonte vers la tête, puis retourne en arrière en s'élargissant très brusquement et occupant toute l'épaisseur du corps sans faire de boucle (fig. 4 A). Région caudale enroulée sur 2 tours de spires et ornée ventralement par une area rugosa constituée de rangées transversales de bâtonnets cuticulaires longitudinaux; sur les flancs, ces rangées s'estompent et la cuticule s'orne de petites perles ; la région postcloacale est également ornée de perles cuticulaires (fig. $4 \mathrm{E}, \mathrm{F}$ et $\mathrm{G}$ ). La queue porte une énorme papille précloacale et 2 rangées ventrolatérales de 5 papilles postcloacales dont la taille diminue vers la pointe caudale. Spicule droit de forme simple et concave; spicule gauche au manche atrophié et à la lame bien développée, effilée vers l'extrémité distale qui est formée par un axe cuticulaire plus long que les ailes étroites qui le bordent (fig. 4, $\mathrm{K}, \mathrm{H}$, I et $\mathbf{J})$.

Dimensions: Corps long de $0,80 \mathrm{~cm}$ et large de $530 \mu$; capsule buccale haute de $5 \mu$; plateau céphalique de $42 \mu / 25 \mu$; anneau nerveux à $150 \mu$ de l'apex; œsophages musculaire et glandulaire longs respectivement de 140 et $900 \mu$; area rugosa longue de $3720 \mu$; bâtonnets de l'area longs de $10 \mu$; espacement entre 2 rangées de bâtonnets large de 8-10 $\mu$; spicule gauche long de $235 \mu$ (lame longue de $192 \mu$ ); spicule droit long de $100 \mu$; queue longue de $68 \mu$.

-2 autres $\hat{o}$ : corps long de 0,88 et $0,94 \mathrm{~cm}$ et large de 440 et $400 \mu$; œsophages musculaire et glandulaire longs de 255-170 $\mu$. et 870-1200 $\mu$; spicules gauche et droit d'un des $2 \hat{o}$ longs de 190 et $140 \mu$. 

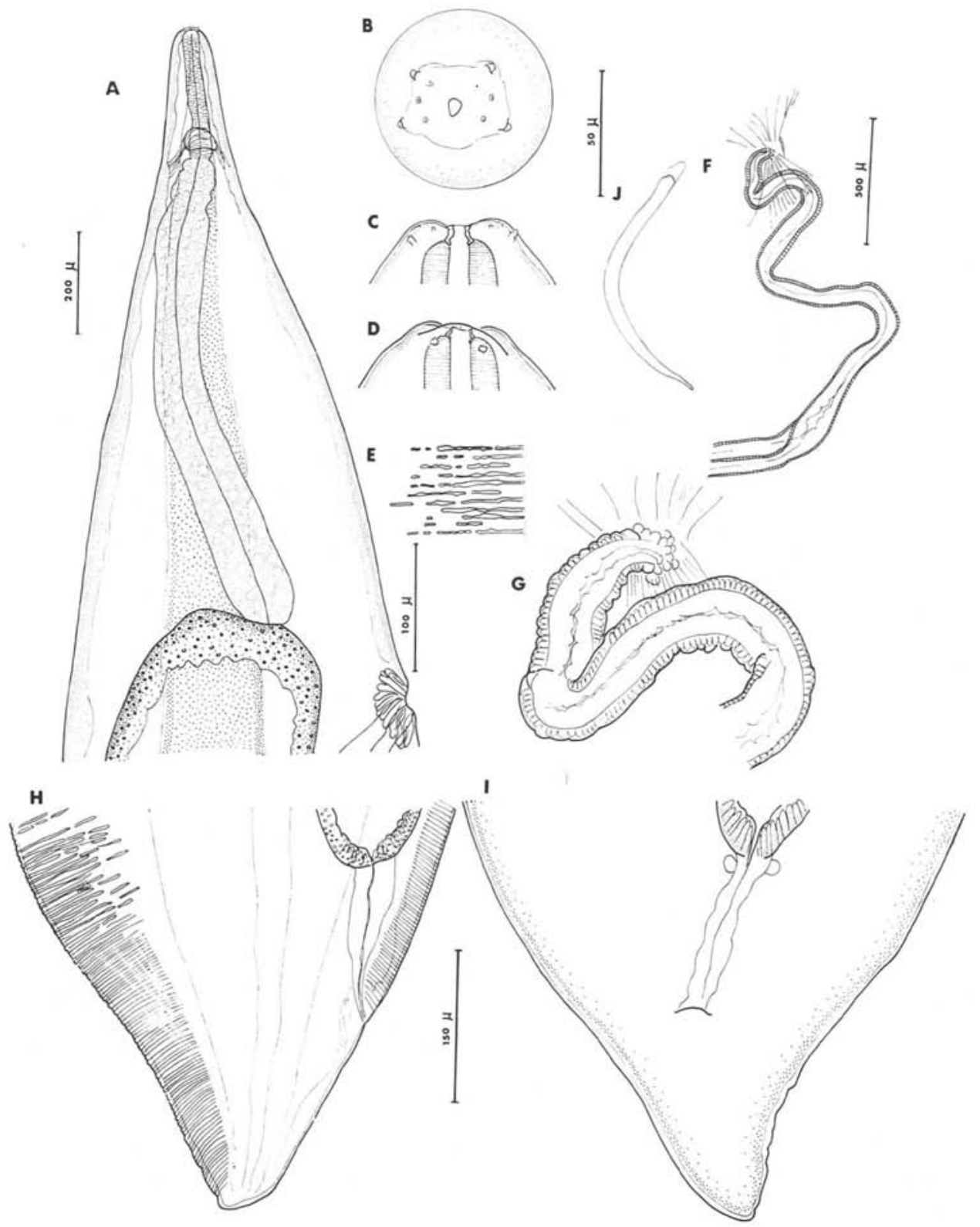

FIG. 3. - Ochoterenella guibei n.sp., ‥ A) Région antérieure, vue latérale. B) Tête, vue apicale. C et D) Idem, respectivement en vue latérale et médiane. E) Ornementation cuticulaire dans la région moyenne du corps. F) Portion impaire de l'ovéjecteur (disséquée). G) Idem, détail de la région proximale. $\mathrm{H}$ et $\mathrm{I})$ Queue, respectivement en vue latérale et en vue ventrale. J) Microfilaire utérine. (A : éch. $200 \mu$; B, C, D, J : éch. $50 \mu$; E : éch. $100 \mu$; F: éch. $500 \mu ; \mathrm{G}, \mathrm{H}, \mathrm{I}$ : éch. $150 \mu$ ). 

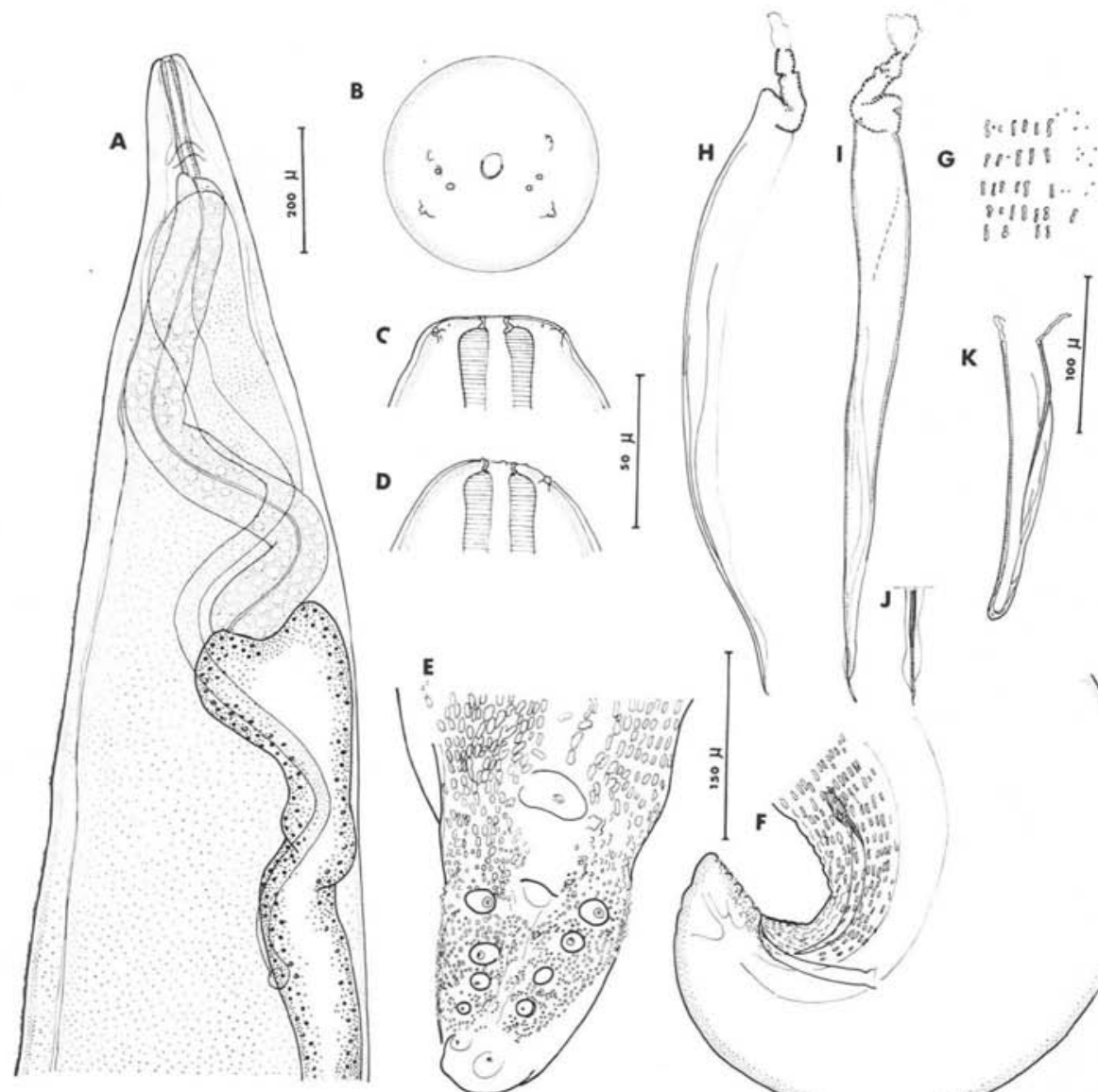

G $81 \cdot 818:+$

10 is 1 in.

$8=1896$
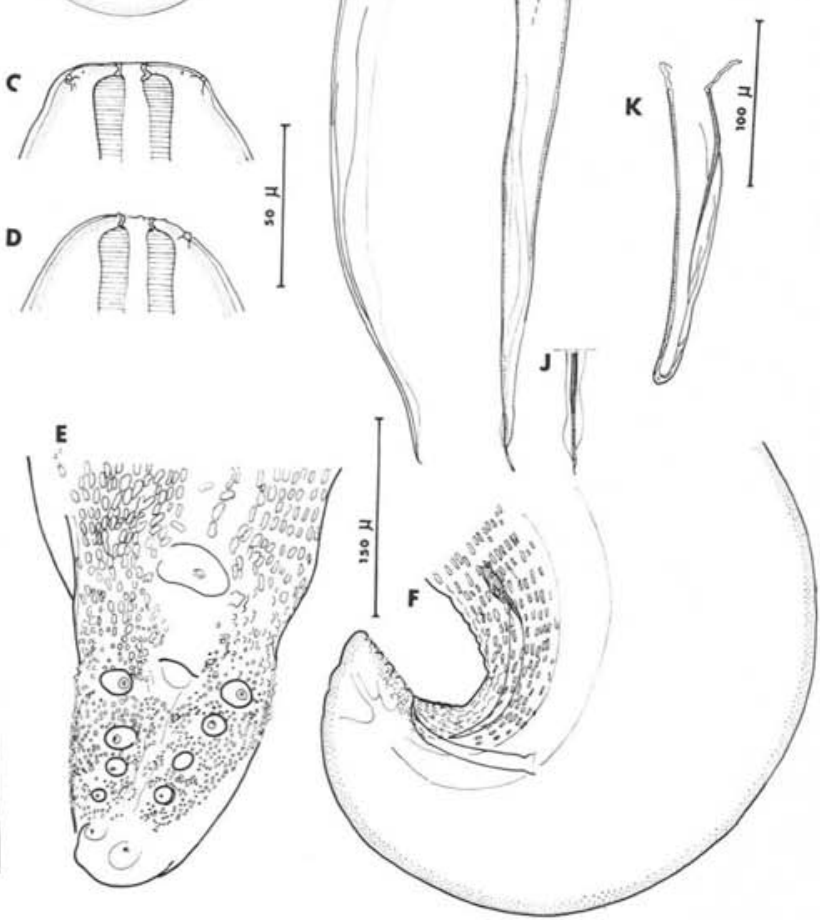

FIG. 4. - Ochoterenella guibei n.sp., ô. A) Région antérieure, vue latérale. B) Tête, vue apicale. $\mathrm{C}$ et $\mathrm{D})$ Idem, respectivement en vues latérale et médiane. E) Queue, vue ventrale. F) Queue, vue latérale. G) Fragment de l'area rugosa dans le plan latéro-ventral. $\mathrm{H}$. et I) Spicule gauche disséqué en vues latérale et ventrale. J) Pointe du spicule gauche, vue ventrale. K) Spicule droit disséqué en vue latéro-ventrale. (A : éch. $200 \mu ; \mathrm{B}, \mathrm{C}, \mathrm{D}, \mathrm{E}, \mathrm{H}$, $\mathrm{I}, \mathrm{J}, \mathrm{K}$ : éch. $50 \mu$; F : éch. $150 \mu ; \mathrm{G}$ : éch. $100 \mu$ ).

Microfilaires (fig. $3 \mathrm{~J}$ ) (extraites de l'utérus) : gaine visible, tête renflée ; région caudale brusquement amincie. Corps longs de 100, 102, 102 et $105 \mu$ sur 3-4 $\mu$ de large.

\section{Discussion.}

Ces Filaires, ayant une vulve située au niveau ou près de l'œsophage et n'ayant ni expansions cuticulaires parabuccales, ni ailes latérales, ne peuvent être rapprochées des autres Filaires malgaches, représentées par le genre Madochotera. 
Les autres Filaires de Batraciens, originaires d'Amérique, d'Afrique et d'Asie, groupées par Schacher dans les Waltonella (= Waltonia Schacher et Crans, 1973 préemployé), ainsi que les 3 espèces sud-américaines décrites ou revues par Travassos (1929) et qu'il n'y a pas lieu d'exclure des Waltonella, ont toutes des ailes caudales qui les distinguent de nos spécimens; en outre, la présence d'expansions cuticulaires parabuccales chez les 3 Waltonella dont la structure céphalique est connue accentue les différences avec nos spécimens.

Il existe par contre une grande ressemblance entre nos Filaires et Ochoterenella digiticauda, parasite de Ranidae mexicains: la tête ne porte pas de formations cuticulaires parabuccales, il n'y a pas d'ailes latérales et le manche du spicule gauche est atrophié ; cette espèce est toutefois distincte par sa plus grande longueur qui lui confère une silhouette beaucoup moins trapue, et par l'ornementation cuticulaire constituée de bosses espacées; l'area rugosa, bien qu'elle n'ait été décrite ni par Caballero (1944) ni par Lent, Freitas et Proença (1946), est représentée sur la figure de la page 88 par Caballero et semble également constituée de bosses.

La $2^{\circ}$ espèce placée dans le genre Ochoterenella, O. papuensis Johnston, 1967, est parasite de Ranidae de Nouvelle-Guinée; la structure céphalique est mal interprétée et ne peut être comparée à celle de nos spécimens; cette espèce de petite taille, à spicule et queue courts comme nos Filaires, est distincte par le corps beaucoup moins large à cuticule lisse, et l'area rugosa qui ne se poursuit pas sur la queue.

Les caractères communs à nos spécimens et à l'espèce type du genre Ochoterenella (absence de formations cuticulaires parabuccales et d'ailes latérales, manche du spicule gauche atrophié) font placer nos spécimens dans ce genre; ils constituent une nouvelle espèce que nous nommons Ochoterenella guibei n. sp.

\section{Waltonella guyanensis n. sp.}

Hôte: Bufo marinus L., Bufonidae.

Localisation: dans le mésentère dorsal.

Provenance: Maripassoula, Guyane française.

MATÉRIEL : 2 o et 6 \%, lot type $300 \mathrm{RL}$.

\section{Description.}

Corps ne présentant pas d'ailes latérales, sauf chez le $\delta^{\star}$ au niveau de la région caudale (fig. 6, I). Bouche grande et arrondie; 2 petites protubérances cuticulaires latérales situées au niveau d'un sillon cuticulaire péribuccal; papilles de la tête disposées schématiquement sur 2 plateaux : 1 plateau postérieur délimité par les papilles céphaliques à pointe articulée, avec bord dorsal et ventral bien marqués, 1 plateau plus antérieur délimité par les papilles labiales externes avec bords latéraux bien 
marqués (fig. 5: B et fig. 6 B, C et D). Capsule buccale courte, difficile à observer, apparemment peu cuticularisée. Esophage nettement divisé présentant une portion glandulaire très longue. Intestin large. Ornementation cuticulaire du corps constituée de rangées de petits bâtonnets rectangulaires (fig. $5 \mathrm{C}$ ), interrompus au niveau des champs latéraux. Cette ornementation, bien visible dans la partie moyenne du corps, s'estompe et devient plus lâche à ses deux extrémités, surtout au niveau de la partie antérieure du corps ; elle commence à devenir visible au niveau du $1 / 7^{\circ}$ antérieur du corps et s'arrête un peu en avant de l'extrémité caudale chez la $q$ et nettement en avant de la queue chez le $\delta$.

Femelle (fig. 5) : vulve étirée latéralement, située peu après le milieu de l'œsophage glandulaire. Musculature de la vulve très développée, constituée par 4 faisceaux attachés au tégument (fig. $5 \mathrm{G}$ ). Ovéjecteur sans sphincter différencié, présentant une partie impaire très longue (fig. $5 \mathrm{G}$ ). Ovaires à extrémité arrondie (fig. $5 \mathrm{~F}$ ). Queue longue, assez large, avec une extrémité plus fine et arrondie (fig. $5 \mathrm{D}$ et E).

Dimensions : Corps long de 2,6 à 4,5 cm $(2,6 \mathrm{~cm}, 3,2 \mathrm{~cm}, 3,3 \mathrm{~cm}, 3,7 \mathrm{~cm}, 4,4 \mathrm{~cm}$, et $4,5 \mathrm{~cm}$ sur respectivement $260 \mu, 290 \mu, 350 \mu, 430 \mu, 450 \mu$ de large).

Pour une $ᄋ$ longue de $4,4 \mathrm{~cm}$, corps large de $450 \mu$ à sa partie moyenne; plateau céphalique long de $78 \mu$ et large de $50 \mu$; ornementation cuticulaire naissant à $5100 \mu$ de l'apex et se terminant à $180 \mu$ de l'extrémité caudale composée de bâtonnets d'environ $5 \mu$ de long sur $1 \mu$ de large et espacés de 4 à $5 \mu$; les bandes de bâtonnets sont espacées de 30 à $35 \mu$; capsule buccale haute de $4 \mu$; anneau nerveux à $200 \mu$ de l'apex ; œsophages musculaire et glandulaire longs respectivement de $310 \mu$ et $1550 \mu$; vulve à $1250 \mu$ de la tête ; ovéjecteur impair long de $2450 \mu$; queue longue de $640 \mu$.

MÂle (fig. 6) : région postérieure enroulée sur 2 à 3 tours de spire ; sur la partie ventrale de la queue et de la région enroulée, area rugosa punctiforme (fig. 6 I et $\mathrm{J}$ ) ; plateau céphalique beaucoup plus saillant que chez la $\$$, car les papilles céphaliques sont très protubérantes (fig. $6 \mathrm{~B}, \mathrm{C}$ et $\mathrm{D}$ ) ; papilles caudales disposées selon la fig. $6 \mathrm{I}$; spicule gauche simple avec lame constituée par une portion proximale membraneuse et une portion distale réduite à un flagelle (fig. $6 \mathrm{G}$ et $\mathrm{H}$ ) ; spicule droit large, beaucoup plus court, et avec 2 petites ailes membraneuses latéro-ventrales (fig. $6 \mathrm{E}$ et $\mathrm{F}$ )

Dimensions: Corps long de 2,1 et $2,5 \mathrm{~cm}$. Pour le $\hat{\delta}$ long de $2,1 \mathrm{~cm}$, corps large de $370 \mu$ dans sa partie moyenne ; ornementation cuticulaire (bâtonnets longs de $4 \mu$ et espacés de 3 à $4 \mu$ ) naissant à $3100 \mu$ de l'apex et se terminant à $650 \mu$ de l'extrémité caudale ; area rugosa débutant à $300 \mu$ en arrière du cloaque et se terminant au niveau de la $2^{\mathrm{e}}$ paire de papilles postcloacales (mesures effectuées sur le $2^{\circ}$ spécimen); plateau céphalique long de $58 \mu$. et large de $37 \mu$; capsule buccale haute de $6 \mu$; anneau nerveux à $200 \mu$ de l'apex; œsophages musculaire et glandulaire longs respectivement de $270 \mu$ et $1625 \mu$; spicule gauche long de $193 \mu$ (manche long de $75 \mu$, parties membraneuse et flagellaire longues respectivement de 76 et $42 \mu$ ) sur $8 \mu$ de large dans sa partie moyenne ; spicule droit long de $121 \mu$ sur $23 \mu$ de large; queue longue de $157 \mu$.

Microfilaire (fig. 51) (extraite de l'utérus): corps long de 130 à $190 \mu$ sur 4 à $5 \mu$ de large. Tête ronde ornée d'un petit crochet et de deux plaques cuticulaires 


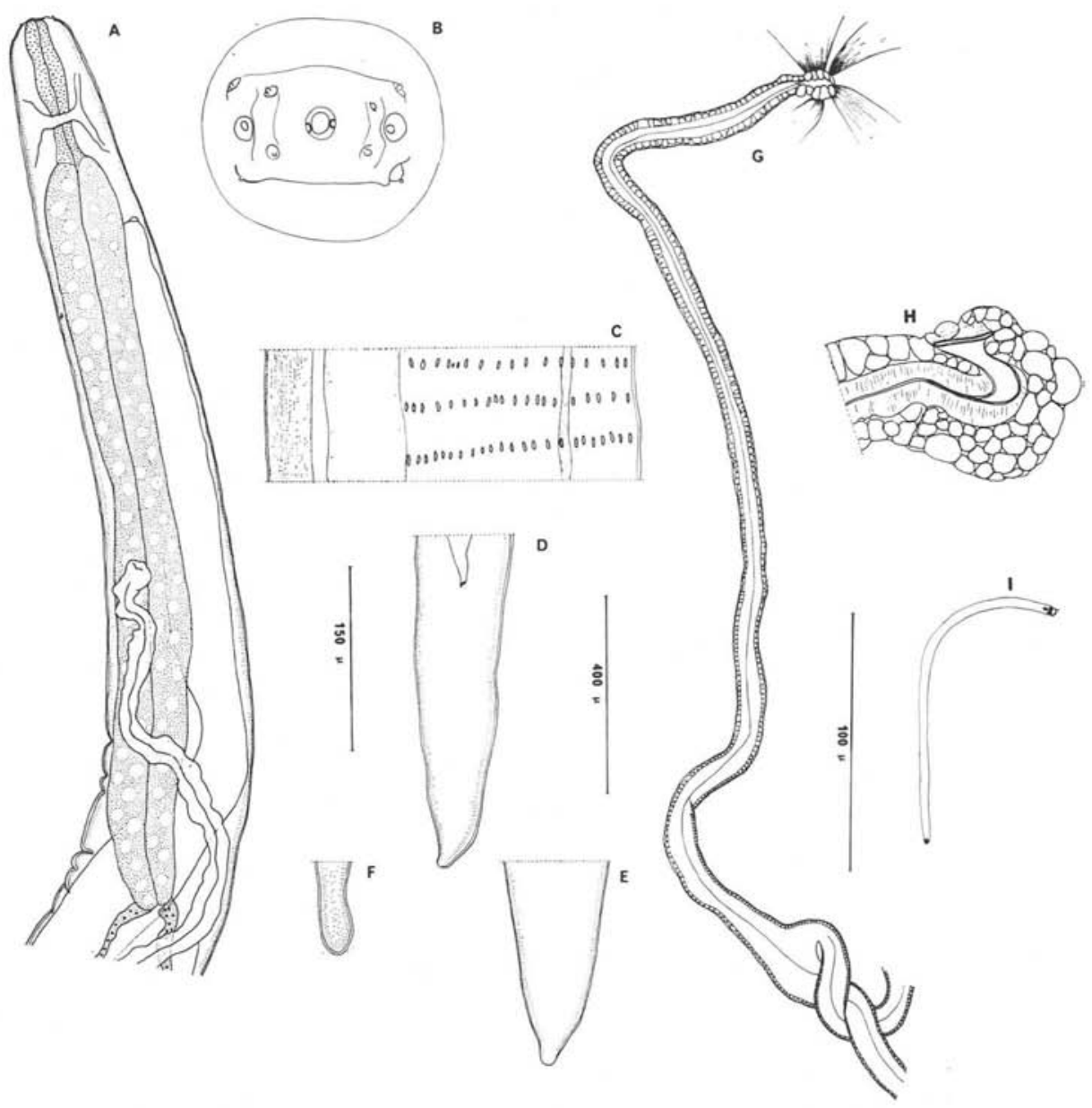

FIG. 5. - Waltonella guyanensis n.sp. ‥ A) Région antérieure, vue ventrale. B) Tête, vue apicale. C) Ornementation cuticulaire, vue latéro-ventrale. D) Région caudale, vue ventrale. E) Idem, vue latérale. F) Extrémité distale d'un ovaire. G) Portion impaire de l'ovéjecteur (disséquée). H) Région vulvaire, vue latérale. I) Microfilaire provenant de l'utérus. (A, D, E et $\mathrm{G}$ : éch. $400 \mu ; \mathrm{C}$ et $\mathrm{F}$ : éch. $150 \mu$; B, H et I : éch. $100 \mu$ ).

subapicaux. Espace céphalique réduit, plus petit que la largeur du corps. Queue entièrement nucléée.

\section{Discussion.}

Par la vulve située au niveau de l'œsophage, ces spécimens se distinguent des Madochotera. Par la présence des formations cuticulaires parabuccales et des ailes latérales chez les $\delta^{\star}$, par la structure normale du spicule gauche, ils s'opposent également aux Ochoterenella. 


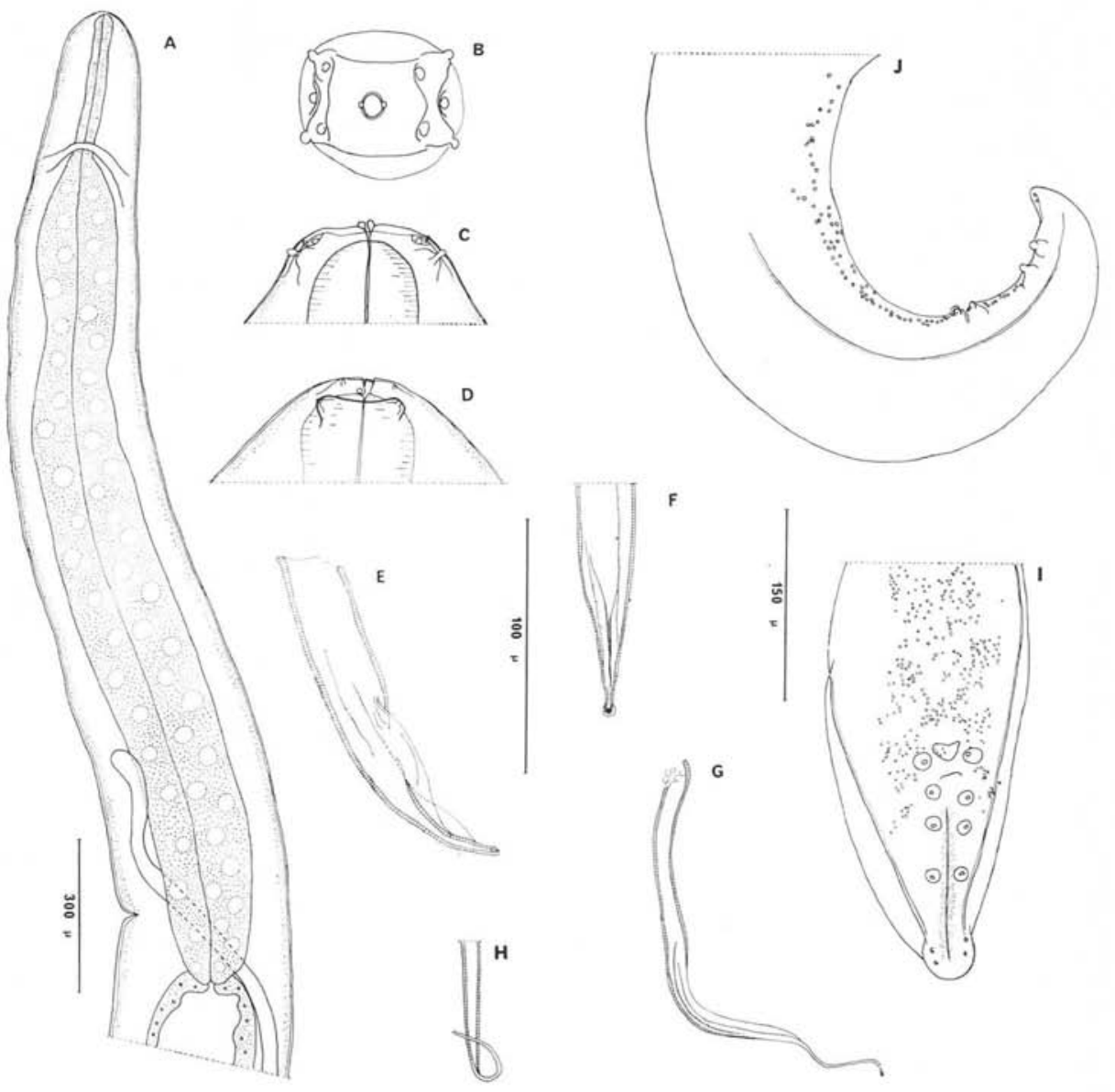

FIG. 6. - Waltonella guyanensis n. sp. o. A) Région antérieure, vue latérale. B) Tête, vue apicale. C) Idem, vue médiane. D) Idem, vue latérale. E) Spicule droit, vue latérale. F) Idem, extrémité distale, vue ventrale. G) Spicule gauche, vue latérale. $\mathrm{H}) \mathrm{Idem}$, extrémité distale, vue ventrale. I) Région caudale et area rugosa, vue ventrale. J) Idem, vue latérale. (A : éch. $300 \mu ; \mathrm{B}, \mathrm{C}, \mathrm{D}, \mathrm{E}, \mathrm{F}, \mathrm{G}$, et $\mathrm{H}$ : éch. $100 \mu$; I et $\mathrm{J}$ : éch. $150 \mu$.).

Ils se rapprochent des nombreuses Filaires de Batraciens à vulve antérieure et ailes caudales; parmi celles-ci, les 3 espèces sud-américaines, parasites de Bufonidae et de Leptodactylidae, W. convoluta (Molin, 1858), W. vellardi (Travassos, 1929) et $W$. scalaris (Travassos, 1929) sont, comme nos spécimens, caractérisées par la présence d'une ornementation cuticulaire du corps dans les 2 sexes, constituée de crêtes longitudinales, mais dont la taille et l'arrangement sont spécifiques et différents de ceux de nos spécimens : 
- W. convoluta a une ornementation distribuée de façon irrégulière, formée d'éléments plus grands que ceux de nos spécimens (6 à $20 \mu$ de long et 4 à $5 \mu$ de large); par ailleurs, le spicule gauche est beaucoup plus grand.

- W. scalaris a une ornementation régulière, mais formée de bâtonnets plus longs $(20 \mu)$ et beaucoup plus serrés dans le sens longitudinal $(5$ à $6 \mu$ contre 20 à $35 \mu)$; le spicule gauche est plus long.

- W. vellardi, parasite de Bufo marinus, comme nos Filaires, a également une ornementation cuticulaire régulière, mais constituée de crêtes plus longues $(16 \mu)$ et disposées de façon beaucoup plus espacée $(0,040$ à $0,112 \mathrm{~mm}$ dans le sens de la longueur et 0,080 à $0,120 \mathrm{~mm}$ transversalement); les spicules sont aussi plus longs.

A côté des espèces décrites ou réétudiées par Travassos, il existe 2 autres Filaires sud-américaines, pour lesquelles la position systématique ne peut être précisée, et qui sont également distinctes de nos spécimens; la Filaire de Bufo marinus décrite en Guyane française par Léger (1918) a une microfilaire terminée par un bourrelet céphalique avec collerette crénelée; la microfilaire de crapaud de Colombie, Filaria columbi Blanchard, 1904) est plus petite (70-90 $\mu)$.

Nos spécimens constituent donc une nouvelle espèce, Waltonella guyanensis n. sp.

\section{Conclusions}

La structure céphalique très particulière et l'area rugosa, maintenant connues chez une douzaine d'espèzes d'origine variée, ainsi que l'ensemble de la morphologie (cf. Introduction), montrent que les genres Waltonella, Madochotera et Ochoterenella sont étroitement liés et éloignés à la fois des Onchocercinae et des Dirofilariinae, auxquels ils étaient rattachés.

Ces genres ne semblent pas davantage devoir être reliés aux autres sous-familles d'Onchocercidae, ni aux Setariidae; leur originalité nous paraît nécessiter la création d'une nouvelle sous-famille :

Waltonellinae, n. subfam. : Onchocercidae à plateau céphalique étiré latéralement avec papilles céphaliques très saillantes et souvent terminées en pointe articulée; généralement, présence de 2 formations cuticulaires parabuccales latérales; cuticule du corps ornée ou lisse ; ailes latérales et caudales très fréquentes ; cavité buccale peu cuticularisée ; œsophage glandulaire toujours bien développé ; queue longue, conique et dépourvue de languettes terminales. Chez le mâle, area rugosa toujours présente, grosses papilles caudales non pédonculées disposées en 2 files régulières submédianes, spicules simples et inégaux; chez la femelle, vulve paraœsophagienne ou plus éloignée de la tête, mais toujours nettement pré-équatoriale, ovéjecteur simple, microfilaire à queue courte entièrement nucléée; parasites de Batraciens Anoures.

Espèce type: Waltonella duboisi (Gedoelst, 1916). 
Il semble que trois groupes, coïncidant avec les anciens genres (et sous-genre), peuvent être distingués dans cette sous-famille: Waltonella, aux formations parabuccales présentes, aux papilles céphaliques munies d'une pointe articulée, aux ailes caudales développées et à la vulve située dans la région œsophagienne, constitue le genre de base à très large distribution ; à Madagascar, il est remplacé par un genre vicariant très proche, Madochotera, distinct par la vulve éloignée de la tête (de 5 à $12 \mathrm{~mm}$ ), les papilles céphaliques dépourvues de pointes articulées et les ailes caudales réduites; Ochoterenella représente un genre plus évolué, ayant perdu les formations parabuccales, les ailes latérales et caudales et dont le spicule gauche a un manche atrophié; il a également une vaste répartition géographique.

Ainsi, selon nous, les Waltonellinae comprennent 3 genres :

- Waltonella Schacher, 1974 (= Waltonia Schacher et Crans, 1973, sous-genre) : formations parabuccales et ailes caudales présentes; ailes latérales très fréquentes ; papilles céphaliques composées d'une base large et d'une pointe articulée; vulve dans la région œsophagienne. Très vaste répartition géographique. Parasite de Ranidae, Bufonidae et Leptodactylidae.

Espèce type : Waltonella duboisi (4) (Gedoelst, 1916) en Afrique.

Autres espèces : W. americana (4) (Walton, 1929), W. ranae (4) (Walton, 1929), W. brachyoptera (4) (Wehr et Causey, 1939), W. dolichoptera (Wehr et Causey, 1939), W. flexicauda (Schacher et Crans, 1973), W. striatus (4) (Ochoterena et Caballero, 1932) en Amérique du Nord; W. convoluta (4) (Molin, 1858), W. vellardi (4) (Travassos, 1929), W. scalaris (4) (Travassos, 1929) et $W$. guyanensis n. sp. en Amérique du Sud; $W$. confusa (Schmidt et Kuntz, 1969) en Asie.

- Madochotera Bain et Brunhes, 1968: formations parabuccales et ailes latérales présentes; ailes caudales réduites; papilles céphaliques dépourvues de pointe articulée; vulve éloignée de la tête $(\geqslant 5 \mathrm{~mm})$. Exclusivement malgache, parasites de Racophoridae.

- Espèce type : $M$. alata Bain et Brunhes, 1968.

Autres espèces : $M$. landauae Prod'hon et Bain, 1973. M. pichoni n. sp. Bain et Brunhes, 1968.

- Ochoterenella Caballero, 1944: pas de formations parabuccales, ni d'ailes latérales et caudales; papilles céphaliques sans pointe articulée; vulve près de l'œsophage; spicule gauche à manche atrophié. Vaste répartition géographique. Parasites de Ranidae, Bufonidae et Racophoridae.

Espèce type: Ochoterenella digiticauda Caballero, 1944, en Amérique Centrale.

Autres espèces: $O$. guibei n. sp. à Madagascar et $O$. papuensis (5) Johnston, 1967 en Asie.

(4) Les espèces munies d'un astérisque ont une structure céphalique inconnue; elles sont néanmoins placées dans le genre Waltonella, car elles ont une vulve antérieure et des ailes latérales et caudales.

(5) La structure céphalique de cette espèce est inconnue. 
Espèces Incertae sedis:

Les espèces Incertae sedis ont été énumérées dans une liste fournie par Wittenberg et Gerichter (1944) et ne peuvent, bien entendu, être placées dans un genre défini.

Toutes les Filaires parasites de Batraciens se répartissent donc en 2 sous-familles: les Waltonellinae $n$. subfam. et les Icosiellinae. Elles ont certains traits primitifs comparables: l'œsophage glandulaire bien développé et la tête ornée de formations cuticulaires saillantes (mais ce sont 2 formations latérales chez les Waltonellinae et 4 épines submédianes chez les Icosiellinae). Ces 2 sous-familles ont en fait des origines très différentes, comme l'attestent l'ensemble de leur morphologie et leur biologie larvaire. Les 5 cycles de Waltonellinae déjà élucidés (Causey, 1939 A et B ; Kotcher, 1941 ; Wittenberg et Gerichter, 1944; Benach et Crans, 1973) et le développement que nous avons obtenu au laboratoire chez Aedes polynesiensis, d'un Waltonella sp., d'un Bufo marinus de Guadeloupe (fig. 7) montrent que le cycle s'effectue chez des moustiques dans le tissu adipeux; le développement est lent; le stade infestant a une queue longue, conique, généralement dépourvue d'expansions cuticulaires. Le cycle d'Icosiella neglecta, le seul connu chez les Icosiellinae, s'effectue chez un Ceratopogonidae et un Psychodidae, dans les muscles thoraciques, en 22 jours; le jeune stade I est si raccourci qu'il prend une forme en amande; la larve infestante a une queue très courte avec 2 mamelons latéraux, une tête avec 2 dents, ventrale et dorsale, et un œsophage très long (Desportes, 1941 et 1942).

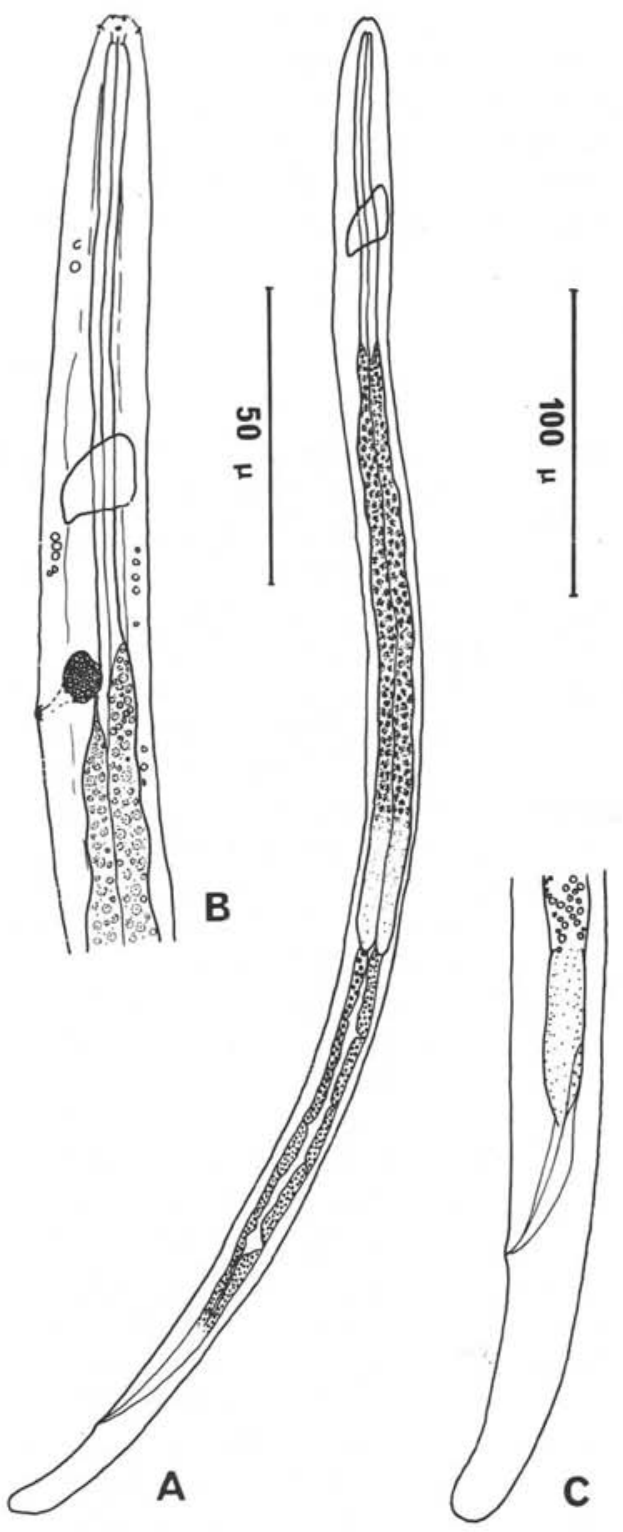

FIG. 7. - Stade infestant de Waltonella $s p$. A) Vue d'ensemble. B) Région antérieure, vue latérale. C) Région postérieure, vue latérale. (A: éch. $100 \mu$; B et $\mathrm{C}$ : éch. $50 \mu$ ). 
Les Waltonellinae ont une très vaste distribution géographique, mais prédominent dans l'hémisphère sud. Les Icosiellinae ne sont connus que par un petit nombre d'espèces, essentiellement dans l'hémisphère nord. Le fait que ces 2 sous-familles ont une grande extension géographique et que leurs représentants sont très homogènes, suggère pour ces Filaires une origine ancienne, vraisemblablement contemporaine de l'apparition des Anoures (Mesozoïque ; cf. Goin et Goin, 1962).

\section{Bibliographie}

ANDERSON (R.C.), 1958. - On the classification of the Filarioidea with special reference to the Filariidae and the Stephanofilariidae. Bull. Soc. Zool., France, 83, 144-159.

-, 1968. - The comparative morphology of cephalic structures in the superfamily Filarioidea (Nematoda). Canad. J. Zool., 46, 181-199.

BAIN (O.) et BRUNHes (J.), 1968. - Un nouveau genre de filaire, parasite de grenouilles malgaches. Bull. Mus. Nat. Hist. nat., (Paris), $2^{\circ}$ sér., 40, 797-801.

Benach (J. L.) et Crans (W. J.), 1973. - Larval development and transmission of Foleyella flexicauda Schacher and Crans, 1973 (Nematoda: Filarioidea) in Culex territans, J. Parasit., 59, 797-800.

Blanchard (R.), 1904. - Commentaire de la note de Montoya y Florès (J. B.), 1904 : De una nueva especie de Filaria en el Sapo de Medellin. Arch. Parasit., 9, 146-148.

Caballero (E.), 1935. - Nematodos parasitos de los batracios de Mexico. III. Cuarta contribucion al conocimiento de la Parasitologia de Rana montezumae. An. Inst. Biol. Univ. Mex., 6, 103-117.

—, 1944. - Estudios helmintologicos de la région oncocercosa de Mexico y de la Republica de Guatemala: Nematoda: La Parte. Filarioidea. I. An. Inst. Biol. Univ. Mex., $15,87-108$.

CAusey (O. R.), 1939 (A). - Aedes and Culex mosquitoes as intermediate hosts of frog. filaria, Foleyella sp. Am. J. Hyg., 29, 79-81.

-, 1939 (B). - Development of larval stages of Foleyella brachyoptera in mosquitoes. Amer. H. Hyg., 30, 69-71.

-, 1939. - Description of three species of frog microfilariae, with notes on staining methods. Amer. J. Hyg., 30, 117-121.

Chabaud (A. G.) in Grasse (P. P.), 1965. - Traité de Zoologie, Némathelminthes, 4, (3). Masson et $C^{\text {ie }}$, édit., Paris, 1497 p.

-, et Anderson (R. C.), 1959. - Nouvel essai de classification des Filaires (super-famille des Filarioidea). II. Ann. Parasit. hum. comp., 34, 64-87.

DESPORTES (C.), 1941. - Nouvelles recherches sur la morphologie et sur l'évolution d'Icosiella neglecta (Diesing, 1851), Filaire commune de la grenouille verte. Ann. Parasit. hum. comp., 18, 46-66.

-, 1942. - Forcipomyia velox Winn. et Sycorax silacea Curtis, vecteurs d'Icosiella neglecta (Diesing), Filaire commune de la Grenouille verte. Ann. Parasit. hum. comp., 19, 58-68. 
Gedoelst (L.), 1916. - Notes sur la faune parasitaire du Congo Belge. Revue Zool. Afr., 5, 1-90.

GoIN (C. J.) et GoIN (O. B.), 1962. - Introduction to Herpetology. W. H. Freeman and Cy, Publ., San Francisco and London, 341 p.

Johnston (M.R. L.), 1967. - Icosiella papuensis n. sp. and Ochoterenella papuensis n, sp, (Nematoda: Filarioidea) from a New Guinea frog, Cornufer papuensis. J. Helminth., 41, 45-54.

Kotcher (E.), 1941. - Studies on the development of frog filariae. Amer. J. Hyg. 34, 3665 .

Leger (M.), 1918. - Microfilaire et filaire du crapaud Bufo marinus L. Bull. Soc. Path. exot., 11, 182-187.

Lent (H.), Freitas (J. F.) et Proenca (M. C.), 1946. - Alguns helmintos de batraquios coelecionados no Paraguai. Mem. Inst. Oswaldo Cruz., 44, 195-214.

Ochoterena (I.) et Caballero (E.), 1932. - Una nueva filaria de las ranas. Ann. Inst. Biol. Univ. Mex., 3, 29-32.

Prod'Hon (J.) et BaIN (O.), 1973. - Madochotera landauae n. sp. nouvelle filaire de grenouille malgache. Bull. Mus. nat. Hist. nat., (Paris) (à parâ̂tre).

SCHACHER (J.F.), 1974. - Waltonella, nom. nov., for subgenus Waltonia (Nematoda: Filarioidea) Schacher et Grans, 1973 preoccupied by Waltonia Davidson 1850 (Brachiopoda). Research note. J. Parasit. (à paraître).

Schacher (J. F.) et Crans (W. J.), 1973. - Foleyella flexicauda sp.n. (Nematoda: Filarioidea) from Rana catesbeiana in New Jersey with a review of the genus and erection of two new subgenera. J. Parasit., 59, 685-691.

Schmidt (G. D.) et KunTz (R. E.), 1969. - Nematodes parasites of Oceanica. VI. Foleyella confusa sp. nov., Icosiella hoogstraali sp. nov. (Filarioidea), and other species from Philippine amphibians. Parasitology, 59, 885-889.

Travassos (L.), 1929. - Filaridés des Batraciens du Brésil. C.R. Soc. Biol., 100, 967-968.

Walton (A. C.), 1929. - Studies on some Nematodes of North American frogs. I. J. Parasit., $15,227-239$.

WeHr (E. E.) et CAusey (O. R.), 1939. - Two new Nematodes (Filarioidea: Dipetalonematidae) from Rana sphenocephala. Amer. J. Hyg., 30, 65-68.

Witenberg (G.) et Gerichter (C.), 1944. - The morphology and life history of Foleyella duboisi with remarks on allied filariids of amphibia. J. Parasit., 30, 245-254. 